\title{
The Reverend Bayes-was he really a prophet?
}

\author{
Nick Freemantle
}

J R Soc Med 2005;98:546

The Reverend Bayes (1702-1761) has become well known for his Theorum, and developed a following of statisticians and their hangers-on who declare themselves to be 'Bayesians'. Does his contribution merit the enthusiasms of his followers? Or is his legacy overhyped?

Bayes did not publish any mathematical work during his lifetime under his own name, and indeed his major attributed work was apparently found by a friend on his desk after his death and remained unpublished until 1764. Although probably at least in large part his own work, and decent work at that, the major objection to Bayes' legacy in the early 21st century is the effect that it has had on those who currently declare themselves to be his followers. This effect has a number of manifestations.

First, Bayesians appear to have an absolute right to disapprove of any conventional approach in statistics without offering a workable alternative - for example, a colleague recently stated at a meeting that '.. it is OK to have multiple comparisons because Bayesians' don't believe in alpha spending'. [Alpha spending is the statistical discipline which governs the interpretation of multiple endpoints in clinical trials. Alpha is 'spent' on the primary outcome, and when this is non-significant it denotes all other statistical tests to be hypothesis generating rather than hypothesis proving.] Other interested parties (such as the pharmaceutical industry) demonstrably do not believe in alpha spending in clinical trials when it suits them (e.g. when the primary outcome of a randomized trial is neutral but some apparently statistically significant results exist with a secondary outcome). The Bayesian trust in sensible people

Professor of Clinical Epidemiology \& Biostatistics, Department of Primary Care and General Practice, Primary Care Clinical Sciences Building, University of Birmingham, Edgbaston, Birmingham B15 2TT, UK

E-mail: N.Freemantle@bham.ac.uk making sensible decisions based upon their prior beliefs in these circumstances seem at best naïve.

Second, Bayesians appear to build an army of straw men - everything it seems is different and better from a Bayesian perspective, although many of the concepts seem remarkably familiar. For example, a very well known Bayesian statistician recently surprised the audience with his discovery of the $P$ value as a useful Bayesian statistic at a meeting in Birmingham.

Third, Bayesians possess enormous enthusiasm for the Gibbs sampler-a form of statistical analysis which simulates distributions based on the data rather than solving them directly through numerical simulation, which they declare to be inherently Bayesian - requiring starting values (priors) and providing posterior distributions (updated priors). However, rather than being of universal application, the Gibbs sampler is really only advantageous in a limited number of situations for complex nonlinear mixed models - and even in those circumstances it frequently sadly just does not work (being capable of producing quite impossible results, or none at all, with depressing regularity). While a step forward, the world is not a different place once populated by Gibbs samplers. And some of those who were involved in the development and evaluation of the Gibbs sampler are arch frequentists (so defined by Bayesians as a pejorative term for all those who work with numbers who are not Bayesians!).

Finally, the social and sartorial objections to Bayesianism are perhaps paramount - sporting a depressingly drab and amorphous uniform of socks and sandals, facial hair and shades of drab green. Coupled with the sellotape repaired glasses and other worldly gleam in the eye, the collective noun for Bayesians is obvious: a posterior.

Bayes (probably) wrote some insightful things, some of which are still relevant now. But once you strip away the hype, his contribution was modest and not the rational basis for a new statistical orthodoxy, or indeed a new religion. 